\title{
BaBar: direct CPV searches
}

\section{Lluïsa-Maria Mir*}

Lawrence Berkeley National Laboratory, USA

E-mail: lmmiralbl.gov

Some recent BaBar measurements of $\mathrm{CP}$-violation charge asymmetries in $\mathrm{B}$ meson decays to charmless hadronic final states are presented.

International Europhysics Conference on High Energy Physics

July 21st - 27th 2005

Lisboa, Portugal

${ }^{*}$ Speaker. 


\section{Introduction}

Direct CP violation or CP violation in decay (DCPV) occurs when the amplitude for a decay and its $\mathrm{CP}$ conjugate process have different magnitudes. It results from the interference among various terms in the decay amplitude, and requires at least two terms with different weak and strong phases. To have large DCPV the two contributing amplitudes should be of the same order, as expected in some charmless B decays with penguin and Cabibbo- (and sometimes color-) suppressed tree amplitudes. It is also interesting to search for DCPV in processes where it is not expected, because new physics in the loop can modify the expectations.

\section{Time-integrated DCVP}

To look for time-integrated DCPV one simply measures the rate difference between a given process and its CP conjugate. It is simple from the experimental point of view and is the only type that can appear in charged decays. It can also be measured on neutral self-tagged decays. DCPV has been searched for in many decays so far [1] but the only process which shows a significant deviation from zero, both in $\mathrm{BaBar}$ and Belle, is $\mathrm{B} \rightarrow \mathrm{K}^{+} \pi^{-}$. This is a self-tagged decay $\left(\mathrm{B}^{0}\right.$ decays to $\mathrm{K}^{+} \pi^{-}$ and $\overline{\mathrm{B}}^{0}$ to $\mathrm{K}^{-} \pi^{+}$). In BaBar a likelihood fit is used to extract the yields and signal and background asymmetries. The measured signal asymmetry is $-0.133 \pm 0.030$ (stat) \pm 0.009 (syst) [2], which corresponds to a $4.2 \sigma$ effect. Fig. 1 shows the results of the fit.

Figure 1: (Left) Background-subtracted distributions of $\Delta E$ for signal $\mathrm{K}^{+} \pi^{-}$(blue, solid) and $\mathrm{K}^{-} \pi^{+}$(red, dashed). (Right) (a) Distribution of $m_{E S}$ enhanced in $\mathrm{K}^{+} \pi^{-}$(blue, solid) and $\mathrm{K}^{+} \pi^{-}$(red, dashed). (b) Asymmetry calculated for ranges of $m_{E S}$.
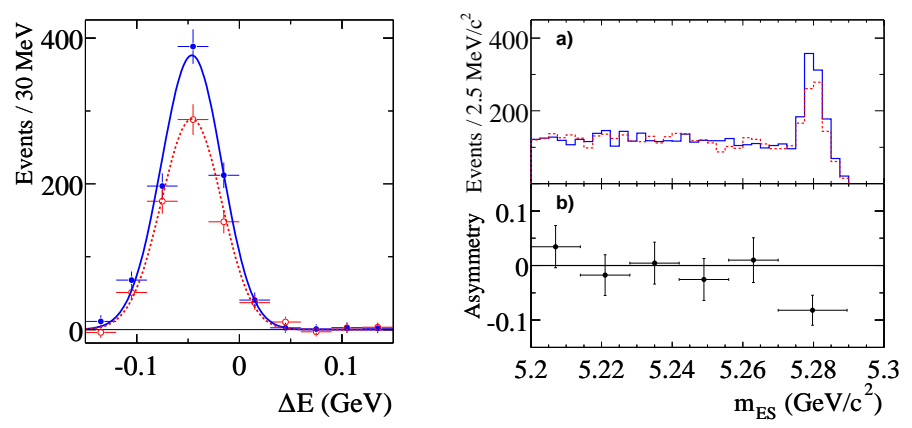

The DCPV expectations for some related decays are different depending on the contributing amplitudes. From naive factorization one would expect the same asymmetry in $\mathrm{K}^{+} \pi^{0}$ as in $\mathrm{K}^{+} \pi^{-}$. Zero asymmetry is expected in $\pi^{+} \pi^{0}$ and possible deviation from zero in $\pi^{0} \pi^{0}$ due to the penguin contribution. Measured asymmetries are shown in Tab. 1. No significant deviation from zero is observed so far.

The Standard Model predicts very small asymmetry for modes involving neutral kaons, these modes being dominated by penguin amplitudes. In agreement with the expectation, no significant asymmetry is measured, as shown in Tab. 2 [3]. 


\begin{tabular}{lccr}
\hline \hline Mode & $\mathrm{n}_{\text {sig }}$ & $\mathrm{BR}\left(\times 10^{-6}\right)$ & \multicolumn{1}{c}{$\mathscr{A}_{\mathrm{CP}}$} \\
\hline $\mathrm{B}^{+} \rightarrow \mathrm{K}^{+} \pi^{0}$ & $682 \pm 39$ & $12.0 \pm 0.7 \pm 0.6$ & $0.06 \pm 0.06 \pm 0.01$ \\
$\mathrm{~B}^{+} \rightarrow \pi^{+} \pi^{0}$ & $379 \pm 41$ & $5.8 \pm 0.6 \pm 0.4$ & $-0.01 \pm 0.10 \pm 0.02$ \\
$\mathrm{~B}^{0} \rightarrow \pi^{0} \pi^{0}$ & $61 \pm 17$ & $1.17 \pm 0.32 \pm 0.10$ & $-0.12 \pm 0.56 \pm 0.06$ \\
\hline \hline
\end{tabular}

Table 1: Measured asymmetries in $\mathrm{K}^{+} \mathrm{pi}^{-}$related decays.

\begin{tabular}{lccr}
\hline \hline Mode & $\mathrm{n}_{\text {sig }}$ & $\mathrm{BR}\left(10^{-6}\right)$ & \multicolumn{1}{c}{$\mathscr{A}_{\mathrm{CP}}$} \\
\hline $\mathrm{B}^{+} \rightarrow \mathrm{K}^{0} \pi^{+}$ & $744_{-36-17}^{+37+21}$ & $26.0 \pm 1.3 \pm 1.0$ & $-0.09 \pm 0.05 \pm 0.01$ \\
$\mathrm{~B}^{+} \rightarrow \overline{\mathrm{K}}^{0} \mathrm{~K}^{+}$ & $41_{-13}^{+15+2}(3.5 \sigma \mathrm{s})$ & $1.5 \pm 0.5 \pm 0.1(<2.4)$ & $0.15 \pm 0.33 \pm 0.03$ \\
$\mathrm{~B}^{0} \rightarrow \mathrm{K}^{0} \overline{\mathrm{K}}^{0}$ & $23_{-7}^{+8} \pm 2(4.5 \sigma \mathrm{s})$ & $1.19_{-0.35}^{+0.40} \pm 0.13$ & \\
\hline \hline
\end{tabular}

Table 2: Measured asymmetries in neutral kaon decays.

A number of three-body charmless B decays have been observed. An example is the final state $\pi^{ \pm} \pi^{ \pm} \pi^{\mp}$ [4], where the $\mathrm{B}^{ \pm} \rightarrow \pi^{ \pm} \pi^{ \pm} \pi^{\mp}$ and $\mathrm{B}^{ \pm} \rightarrow \rho^{0}(770) \pi^{ \pm}$branching fractions, among others, are measured by means of a Dalitz-plot analysis. The summary of branching fractions and charge asymmetries is shown in Tab. 3 .

\begin{tabular}{lccc}
\hline \hline \multicolumn{1}{c}{ Mode } & $\mathrm{BR}\left(10^{-6}\right)$ & $90 \% \mathrm{UL}$ & $\mathscr{A}_{C P}$ \\
\hline $\mathrm{B}^{ \pm} \rightarrow \pi^{ \pm} \pi^{ \pm} \pi^{\mp}$ total & $16.2 \pm 1.2 \pm 0.9$ & - & $-0.7 \pm 7.7 \pm 2.5$ \\
\hline$\rho^{0}(770) \pi^{ \pm}, \rho^{0}(770) \rightarrow \pi^{+} \pi^{-}$ & $8.8 \pm 1.0 \pm 0.6_{-0.7}^{+0.1}$ & - & $-7.4 \pm 12.0 \pm 3.4_{-4.4}^{+0.6}$ \\
$\rho^{0}(1450) \pi^{ \pm}, \rho^{0}(1450) \rightarrow \pi^{+} \pi^{-}$ & $1.0 \pm 0.6 \pm 0.2 \pm 0.2$ & $<2.3$ & $+15.5 \pm 62.1 \pm 7.9_{-1.0}^{+0.4}$ \\
$\mathrm{f}_{0}(980) \pi^{ \pm}, \mathrm{f}_{0}(980) \rightarrow \pi^{+} \pi^{-}$ & $1.2 \pm 0.6 \pm 0.1 \pm 0.4$ & $<3.0$ & $-49.5 \pm 53.7 \pm 4.9_{-2.9}^{+3.7}$ \\
$\mathrm{f}_{2}(1270) \pi^{ \pm}, \mathrm{f}_{2}(1270) \rightarrow \pi^{+} \pi^{-}$ & $2.3 \pm 0.6 \pm 0.2 \pm 0.3$ & $<3.5$ & $-0.4 \pm 24.7 \pm 2.8_{-1.6}^{+0.4}$ \\
$\mathrm{~B}^{ \pm} \rightarrow \pi^{ \pm} \pi^{ \pm} \pi^{\mp}$ nonresonant & $2.3 \pm 0.9 \pm 0.3 \pm 0.4$ & $<4.6$ & $+8.0 \pm 41.2 \pm 6.5 \pm 2.4$ \\
$\chi_{\mathrm{c}^{0}} \pi^{ \pm}, \chi_{\mathrm{c}^{0} \rightarrow \pi^{+} \pi^{-}}$ & - & $<0.3$ & - \\
$\mathrm{f}_{0}(1370) \pi^{ \pm}, \mathrm{f}_{0}(1370) \rightarrow \pi^{+} \pi^{-}$ & - & $<3.0$ & - \\
$\sigma \pi^{ \pm}, \sigma \rightarrow \pi^{+} \pi^{-}$ & - & $<4.1$ & - \\
\hline \hline
\end{tabular}

Table 3: Summary of average branching fraction and charge asymmetry results. The first uncertainty is statistical, the second is systematic, while the third is model-dependent.

\section{Time-dependent DCVP}

In addition to differences of rates, one can look at the time-dependent asymmetry between $\mathrm{CP}$-conjugated neutral processes:

$$
a_{f_{C P}}(t)=\frac{2 \operatorname{Im} \lambda_{f_{C P}}}{1+\left|\lambda_{f_{C P}}\right|^{2}} \sin (\Delta m t)+\frac{1-\left|\lambda_{f_{C P}}\right|^{2}}{1+\left|\lambda_{f_{C P}}\right|^{2}} \cos (\Delta m t)=-S_{f_{C P}} \cdot \sin (\Delta m t)+C_{f_{C P}} \cdot \cos (\Delta m t)
$$


The coefficient in front of the cosine term, $C_{f_{C P}}$, is different from zero if the module of $\lambda_{f_{C P}}$, which includes the ratio of CP-conjugated amplitudes, is different from one. $C_{f_{C P}}$ different from zero will be then an indication of DCPV.

There is no evidence of DCPV on B decays to $\pi^{+} \pi^{-}$[5]. Fig. 2 shows the number of events as a function of time for $\mathrm{B}^{0}$ and $\overline{\mathrm{B}}^{0}$ tags, and the raw asymmetry. The measured asymmetry coefficients are $\mathrm{S}_{\pi^{+} \pi^{-}}=-0.30 \pm 0.17$ (stat) \pm 0.03 (syst) and $\mathrm{C}_{\pi^{+} \pi^{-}}=-0.09 \pm 0.15$ (stat) \pm 0.04 (syst)

Figure 2: Distributions of the decay-time difference $\Delta t$ for $\pi^{+} \pi^{-}$enhanced events.

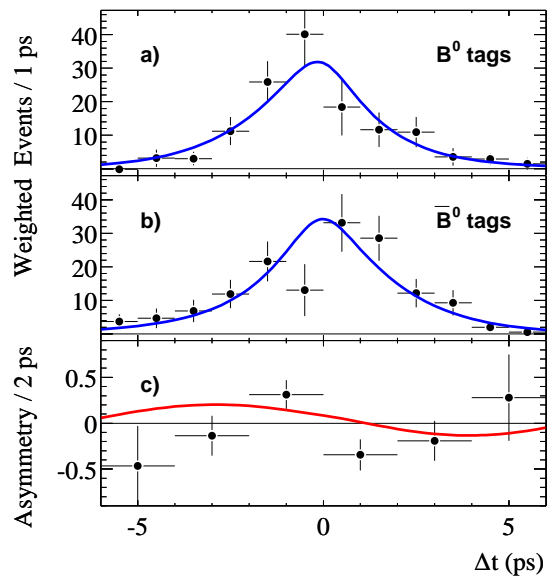

Another interesting measurement is $\mathrm{B} \rightarrow \rho \pi$. QCD factorization predicts no DCPV in this decay. This is a difficult analysis because $\rho^{+} \pi^{-}$is not a CP-eigenstate and there are four flavourcharge configurations to be considered. The approach taken by Babar is to use the full Dalitzplot, so that interferences are taken into account, and to assume that the three resonances $\rho^{0}$, $\rho^{+}$and $\rho^{-}$are dominant [6]. The fact that the final states are not CP-eigenstates complicates the interpretation of the fit results. Because of that the fitted parameters are transformed into the more intuitive quantities $\mathscr{A}_{\rho \pi}^{+-}$and $\mathscr{A}_{\rho \pi}^{-+}$, which involve diagrams where the $\rho$ or the $\pi$ are emitted by the $\mathrm{W}$ boson. The measured values are $\mathscr{A}_{\rho \pi}^{+-}=-0.21 \pm 0.11$ (stat) \pm 0.04 (syst) and $\mathscr{A}_{\rho \pi}^{-+}=-0.47 \pm_{-0.15}^{+0.14}$ (stat) \pm 0.06 (syst). The significance of this observation, including systematic errors, is at the $2.9 \sigma$ level. Fig. 3 shows the confidence level contours for the direct CP asymmetries.

\section{Conclusions}

Direct CP violation in time-integrated measurements is observed in $\mathrm{K}^{+} \pi^{-}$decays. No other significant signal of DCPV has been observed yet, although the uncertainties are still large at present and more data is needed to have conclusive results. There is a 2.9 standard deviation evidence of DCPV in B decays to $\rho \pi$ whereas there is not such an evidence in B decays to $\pi^{+} \pi^{-}$ so far. With more data and improved experimental errors one will be able soon to test whether or not there is large $\mathrm{CP}$ violation in many channels. 
Figure 3: Confidence level contours for the DCPV asymmetries $\mathscr{A}_{\rho \pi}^{+-}$versus $\mathscr{A}_{\rho \pi}^{-+}$. The shaded areas represent $1 \sigma, 2 \sigma$ and $3 \sigma$ contours respectively.

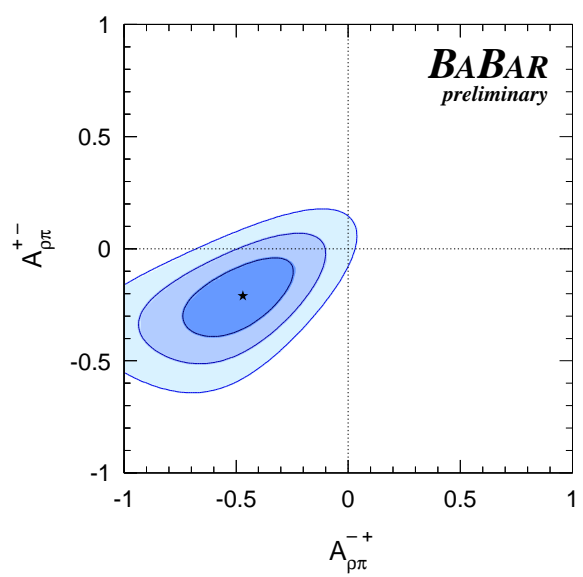

\section{References}

[1] http://www.slac.stanford.edu/xorg/hfag/rare/leppho05/charmless/index.html

[2] B.Aubert et al. BaBar Collaboration. Phys. Rev. Lett. 93, 131801 (2004).

[3] B.Aubert et al. BaBar Collaboration. Phys. Rev. Lett. 95, 221801 (2005).

[4] B.Aubert et al. BaBar Collaboration. Phys. Rev. D 72, 052002 (2005).

[5] B.Aubert et al. BaBar Collaboration. Phys. Rev. Lett. 95, 151803 (2005).

[6] B.Aubert et al. BaBar Collaboration. hep-ex/0408099 